\title{
REDUCTION OF EFFECTS OF INACTIVE ARRAY ELEMENTS IN PHASE ABERRATION CORRECTION
}

\author{
M. Karaman, H. Köymen, A. Atalar, and M. O'Donnell ${ }^{\dagger}$ \\ Department of Electrical and Electronics Engineering \\ Bilkent University, Ankara, 06533 TURKEY \\ $\dagger$ Department of Electrical Engineering and Computer Science \\ University of Michigan, Ann Arbor, MI 48109-2122, USA
}

\begin{abstract}
Phase aberration correction based on time delay estimation via minimization of sum of absolute difference (SAD) between radio frequency (RF) signals of neighboring elements is studied in the presence of missing elements. To examine the influence of inactive elements, phase estimation error is measured for various combinations of different number of missing elements, aberration level, and SNR. The measurements are performed on an experimental RF data set. Aberration delays of missing elements are interpolated using the phase estimate between the nearest active elements. The B-scan images are reconstructed for qualitative examination.
\end{abstract}

\section{INTRODUCTION}

Phase errors induced by tissue inhomogeneities critically affect the image resolution of phased array ultrasound scanners. Various techniques had been developed to estimate and correct phase aberrations $[1,2,3]$. Time delay estimation via minimization of SAD between the sampled RF echo signals of neighbor elements has been shown to perform well for accurate phase estimation with relatively high computational efficiency [5]. Accuracy of the estimation is seriously degraded, however, by the presence of missing elements [4].

Detection of differential delays between missing elements is not possible in aberration correction based on time delay estimation on the neighboring elements. To obtain the complete aberration pattern for proper phasing of whole aperture, the differential delays of missing elements can be interpolated using the phase estimate between the nearest active elements next to missing elements. However, accuracy of phase estimation between two RF signals is limited by level of their covariance [6]. The covariance depends on not only the spatial distance, but it is also critically influenced by aberration level and SNR of RF signals.

To examine the accuracy performance of aberration correction with missing elements, phase estimation error have been measured for different combinations of the spatial distance, aberration level, and SNR. The measurements were performed on a $3.3 \mathrm{MHz}$ 64-element phased array experimental RF data set. Dependence of the measurements on bit density of RF data is examined in the limiting case by using one bit word length for SAD computation. Additionally, B-scan images of a phantom were reconstructed with missing elements for qualitative test.

\section{ABERRATION CORRECTION}

Phased array adaptive beamformers involve estimation of phase distortions across the array, and its compensation using the estimate. The compensation is easily realized by updating focusing delays using the estimated delays. One way is to estimate aberration delays is to measure relative time shifts between neighboring elements. The final element delays, then, can be obtain by unwrapping the measured differential delays [1].

Computational requirements of cost function used in the time delay estimation, limit the computational efficiency of estimation. The SAD can be used as a computationally simple cost function, and is defined as:

$$
E_{m, n}(\boldsymbol{k}, \boldsymbol{u}, \boldsymbol{\alpha})=\sum_{i=1}^{M}\left|S_{m}\left(i+u_{m}\right)-S_{n}\left(i+k+u_{n}\right)\right|
$$

where $S_{n}(i)$ and $u_{n}$ correspond to the $i$ th sample of the signal, and the aberration delay for the $n$th element, respectively. The signals $S_{m}$ and $S_{n}$ are defined as:

$S_{m}=X_{m}+c_{m} W_{1} \quad S_{n}=X_{n}+c_{n} W_{2}$ 
where $X_{m}$ and $X_{n}$ are the noise free RF signals, $W_{1}$ and $W_{2}$ are the uncorrelated Gaussian random noise having the same bandwidth with the RF signals, and $c_{m}$ and $c_{n}$ are the coefficients used to obtain different SNR levels. The best match of $S_{m}$ and $S_{n}$ results in the minimum SAD value. Thus, the value of $k$ which minimizes the SAD corresponds to the time delay difference between $S_{m}$ and $S_{n}$. In the limiting case, the SAD expression can be simplified as:

$D_{m, n}(k, u, \alpha)=\sum_{i=1}^{M} x_{m}\left(i+u_{m}\right) \oplus x_{n}\left(i+k+u_{n}\right)$

where $x_{n}(i)$ is the the one bit representation of $S_{n}(i)$, and $\oplus$ is the bitwise logic EXCLUSIVE-OR operation.

For comparison purpose, the time-delay estimation using normalized cross-correlation as a cost function is considered:

$C_{m, n}(k, u, \alpha)=$

$\frac{\sum_{i=1}^{M}\left\{S_{m}\left(i+u_{m}\right)-\bar{S}_{m}\right\}\left\{S_{n}\left(i+k+u_{n}\right)-\bar{S}_{n}\right\}}{\sqrt{\sum_{i=1}^{M}\left\{S_{m}\left(i+u_{m}\right)-\bar{S}_{m}\right\}^{2} \sum_{i=1}^{M}\left\{S_{n}\left(i+k+u_{n}\right)-\bar{S}_{n}\right\}^{2}}}$

where the $\bar{S}_{n}$ is the mean value of $S_{n}($.$) computed over$ the corresponding window. The best match of two signals is achieved at the maximum correlation value where the maximizing parameter, $k$, corresponds to the time delay between $S_{m}$ and $S_{n}$.

To obtain the aberration delays, the time delay estimation outlined above must be repeated for all neighboring element pairs. The estimate of resultant aberration delay time, $\tau_{n}$, for the $n$th element is obtained by the cumulative summation:

$\tau_{n}=\sum_{j=2}^{n} \Delta \tau_{j, j-1}$

where $\Delta \tau_{j, j-1}$ is the differential delay between the $j$ th and $(j-1)$ th elements.

Presence of missing elements causes loss of corresponding differential delays. Attempts to obtain the complete aberration pattern with lost differential delays result in forming two phase patterns over the subapertures on two sides of inactive elements. While elements of each subaperture are phased properly, the relative phasing of two subapertures with respect to each other may not be correct. This can cause significant degradation in image resolution. To handle this problem, the relative phase between two nearest operable elements on two sides of inactive elements is estimated, and it is evenly distributed over the missing elements [4]. If the elements between $i$ and $i+r$ are inactive, then the differential delay between the $n$th and $(n-1)$ th elements, $\Delta \tau_{n, n-1},(i<n \leq i+r)$ is obtained as:

$\Delta \tau_{n, n-1}=\frac{\Delta \tau_{i+r, i}}{r}$

\section{MEASUREMENTS}

To test influence of missing elements on aberration correction, various measurements are performed using an actual RF data set acquired from a standard graphitegel AIUM resolution phantom with a conventional 3.3 MHz, 64-element phased array [1]. The RF Ascans were recorded for every possible combination of transmitter and receiver elements, using 10-bit, $17.76 \mathrm{MHz}$ analog to digital conversion. All measurements and image reconstruction are carried out by digitally processing the recorded data to emulate the operation of a real-time adaptive digital imaging system.

To reduce the phase quantization effects, the sampling rate of data is increased by 8 times using digital interpolation resulting in a phase accuracy of $2 \pi / 43$. For all measurements, a fixed transmit focus at $80 \mathrm{~mm}$, and dynamic receive focusing are employed. Correlation and SAD functions are calculated on RF signals from diffuse scatterers within a $20 \mathrm{~mm}$ axial window located at $60 \mathrm{~mm}$ away from the aperture.

\section{Phase Estimation Error}

The time delay estimation error vs rms aberration over the array, $r m s\{u\}$, and spatial distance, $r$, is measured for correlation and 1-bit SAD functions. The measured error is the residual rms phase error between the original and estimated relative aberration delays. Note that the number of missing elements is equal to $r-1$ for $r \geq 1$. Since the measurements are performed on the different element pairs on the array, the error of each measurement is first averaged over the pairs with the same spatial separation:

$\Delta \phi_{r}^{u}=\sqrt{\frac{1}{N-r} \sum_{m=1}^{N-r}\left\{\Delta \tau_{m, m+r}-\left(u_{m}-u_{m+r}\right)\right\}^{2}}$

Then, the rms of this averaged error and its standard deviation over five different speckle data are obtained, denoted by $r m s\left(\Delta \phi_{r}^{u}\right)$.

To obtain the results, the relative phase differences between the element pairs are estimated by the time delay estimation with $\alpha=\infty$ in Eqs. 3 and 4, and then the above error computations are carried out. The estimation is repeated for all element pairs separated by $r$ elements, and for different $r m s\{u\}$ values.

Fig. 1 depicts the $r m s\left(\Delta \phi_{r}^{u}\right)$ vs $r$ and $r m s\{u\}$ for the correlation and 1-bit SAD. The isopotential contour 

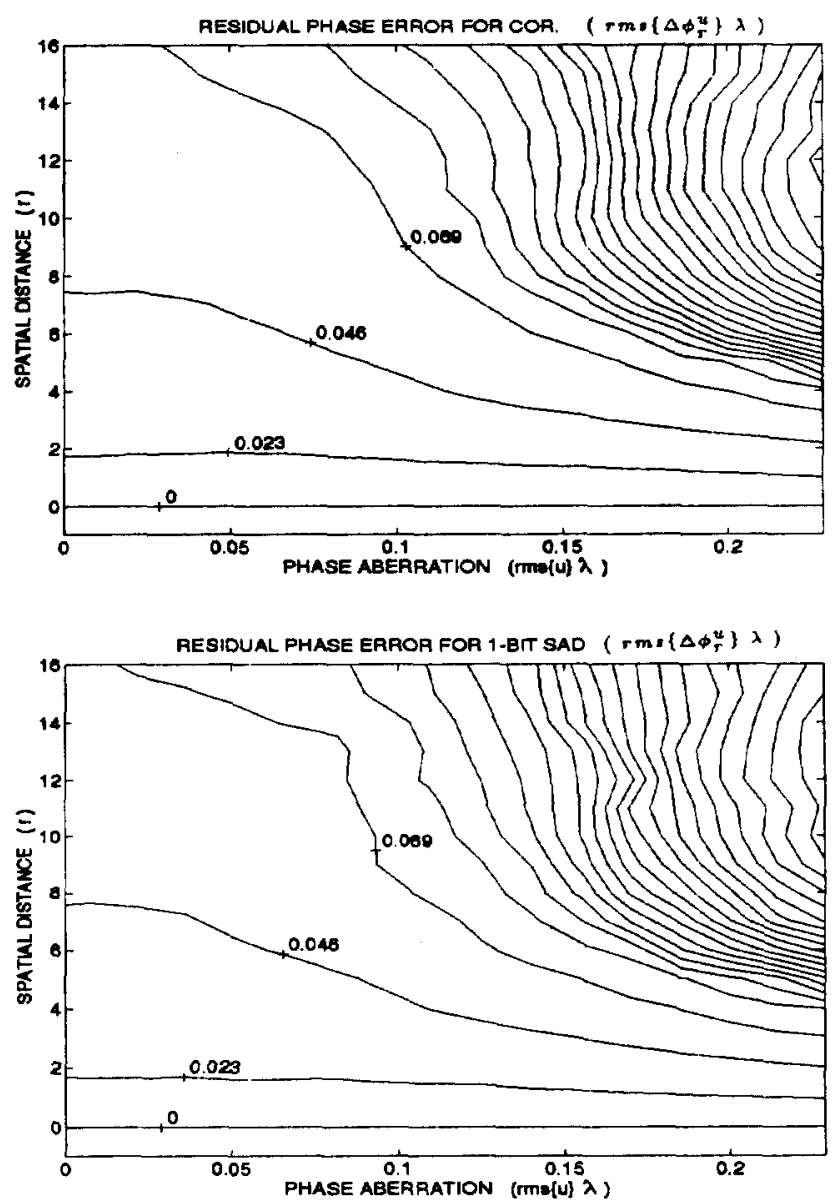

Figure 1: Isopotential contours of the residual rms phase error vs the spatial distance and rms phase aberration.

values shown on the figures are given in fractions of a wavelength, and the contours are equally spaced. The error distributions for correlation and 1-bit SAD are almost the same. The error for the combinations of number of missing elements and rms aberration levels under 0.069 contour can be tolerable for time delay estimation (one quantization level is $0.023 \lambda$ ). In general, the phase estimation error is acceptable for missing elements up to 10 percent of overall aperture with rms aberration up to $0.15 \lambda$. The measured standard deviations indicate that while the results below the 0.069 contour are stable, the error measurements vary significantly with different speckle data for measurements above this error, and hence are not stable.

\section{Noise Performance}

To test noise performance of aberration estimation using correlation and 1-bit SAD, phase estimation error is measured for different SNR levels and spatial
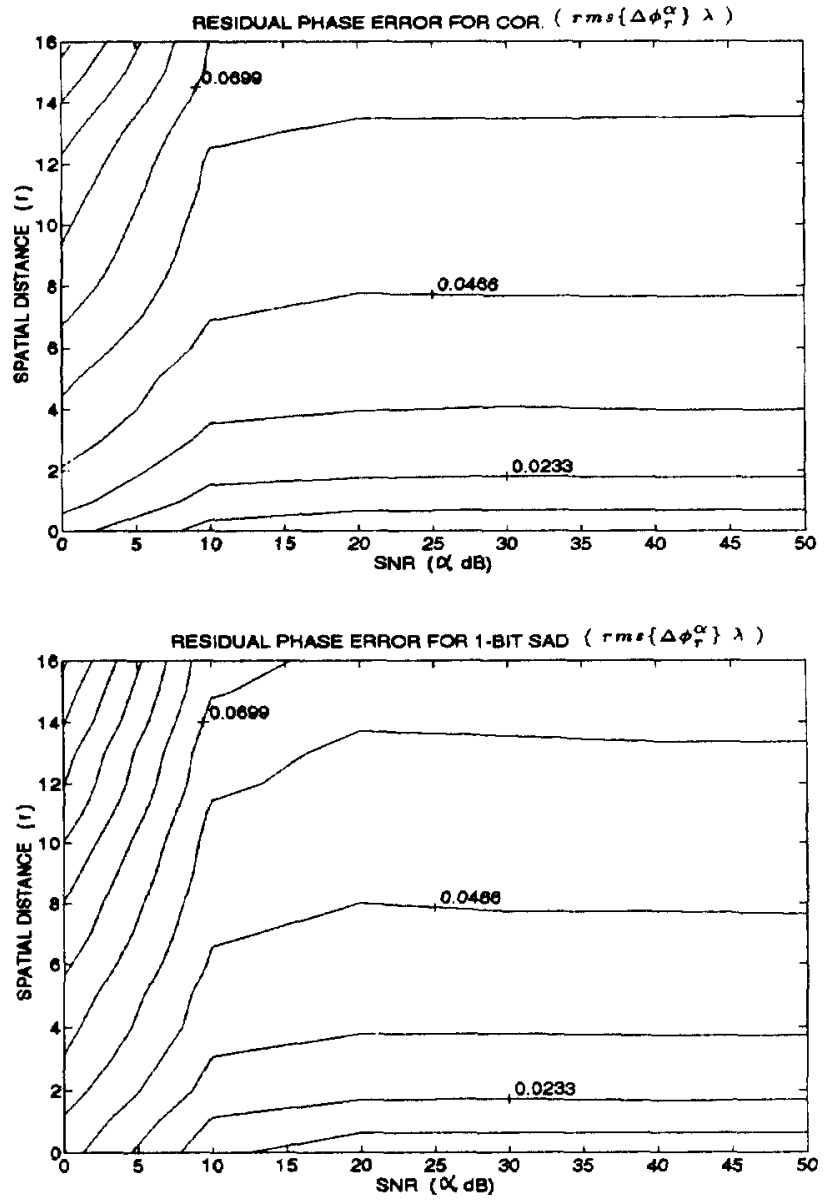

Figure 2: Isopotential contours of the residual $\mathrm{rms}$ phase error vs the spatial distance and SNR.

distances (see Fig. 2). To obtain these results, the noisy signals, $S_{m}$ and $S_{n}$ (Eq. 2), are generated by proper setting of the coefficients, $c_{m}$ and $c_{n}$. The rms and standard deviation computations over five speckle data are carried out as similar to those in the previous subsection. Note that, in the phase estimation measurements, the cost functions of Eqs. 3 and 4 are computed with no aberration, $u_{i}=0$, and hence the resulting error is a function of only the spatial distance, $r$, and SNR, $\alpha$. The resultant residual $r m s$ error is denoted as $r m s\left\{\Delta \phi_{r}^{\alpha}\right\}$.

It must be noted that, in the measurements, it is assumed that the original data is noiseless. In fact the original data is real data and contains noise. Therefore the generated noise is actually an additive noise on the signal. Hence, the SNR values reported in this work are lower than the actual SNR values.

The residual phase error measurements vs SNR and spatial distance for correlation and 1-bit SAD are given in Fig. 2. The correlation and 1-bit SAD functions, in 


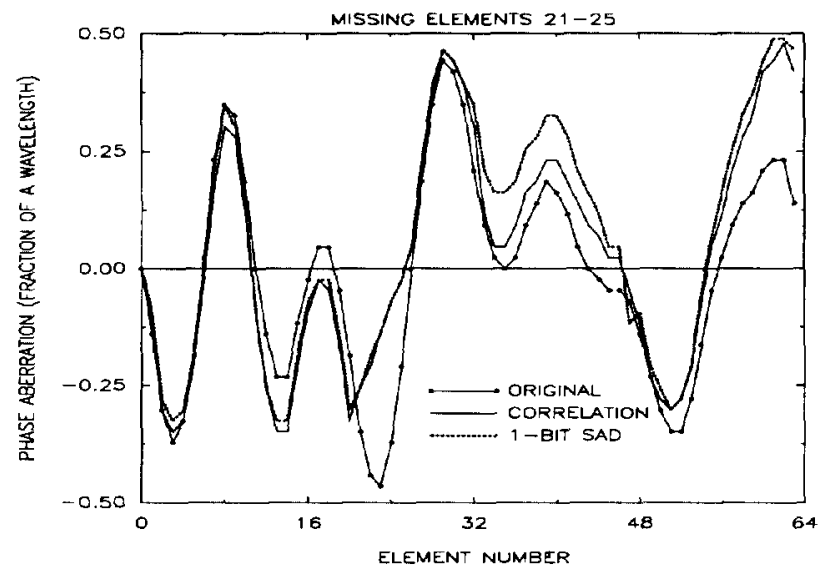

Figure 3: Original and estimated aberration patterns.

general, show similar performance in phase estimation for different SNR levels. However, the error of 1-bit SAD is slightly higher than that of correlation at very low SNR. The measured standard deviations indicate that the results are quite stable for different speckle data with SNR higher than $10 \mathrm{~dB}$.

\section{B-SCAN IMAGES}

For qualitative testing, the sector images of the phantom are reconstructed with missing elements and aberration. Each image shown in Fig. 4 shows a section of the phantom, and has a size of about $2.5 \mathrm{~cm} \times 2.5 \mathrm{~cm}$. In the reconstruction, dynamic receive focusing is employed, and transmit focus is fixed at $80 \mathrm{~mm}$. The images are displayed using only a logarithmic compression over a $50 \mathrm{~dB}$ dynamic range.

For each corrected image, the transmit and receive delays are updated using the aberration pattern estimated from diffuse scatterers with missing elements 21-25. The window length used for correlation and 1bit SAD is $20 \mathrm{~mm}$. The missing elements and phase distortion are employed in both transmit and receive beamforming. The original and estimated aberration patterns shown in Fig. 3 ar $\geqslant$ used for electronic phase distortion and compensation, respectively.

Comparison of the control, aberrated, and corrected images shown in Fig. 4 indicates that the degradation due to aberration is significantly eliminated even with the presence of missing elements. The corrected images are very similar which clearly depicts the comparable performance of correction schemes based on 1-bit SAD and correlation. Slight difference between the control and corrected images is due to unperfect phase error compensation arising from accumulation of small estimation errors.

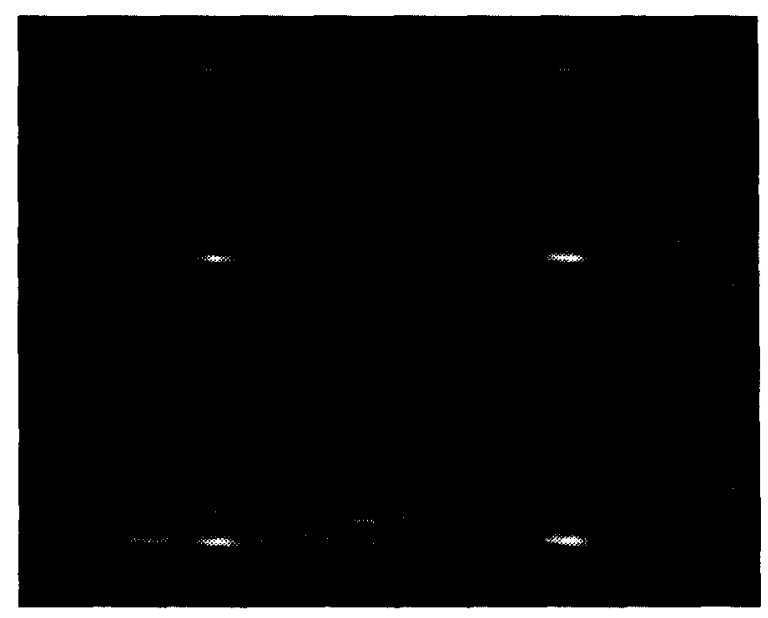

Figure 4: B-scan images: a) control b) aberrated, and corrected using c) correlation and d) 1-bit SAD.

\section{CONCLUSION}

Influence of missing elements in aberration correction based on time delay estimation on neighboring elements is investigated through the measurements performed on the experimental data. The measurements show that 1-bit SAD can be effectively used for correction of phase distortions with adjacent missing elements up to $10 \%$ of a 64 -element phased array. Weak accuracy performance of 1-bit SAD at very low SNR can be improved by averaging the estimates over a number of beams, and/or increasing the estimation window [5].

\section{ACKNOWLEDGMENT}

This work is supported in part by Turkish Scientific and Research Council, TUBITAK.

\section{References}

[1] S. W. Flax and M. O'Donnell, "Phase-aberration correction using signals from point reflectors and diffuse scatterers: basic principles," IEEE Trans. Ultrason. Ferroelec. Freq. Contr., pp. 758-767, Nov. 1988.

[2] D. Zhao and G. E. Trahey, "Comparisons of image quality factors for phase aberration correction with diffuse and point targets," IEEE Trans. Ultrason. Ferroelec. Freq. Contr., pp. 125-132, Mar. 1991.

[3] M. Fink, "Time reversal of ultrasonic fields - Part I: basic principles", IEEE Trans. Ultrason. Ferroelec. Freq. Contr., pp. 555-566, Sep. 1992.

[4] M. O'Donnell and W. E. Engeler, "Correlation-based aberration correction in the presence of inoperable elements," IEEE Trans. Ultrason. Ferroelec. Freq. Contr., pp. 700-707, Nov. 1992.

[5] M. Karaman, A. Atalar, H. Köymen, and M. O'Donnell, "A phase aberration correction method for ultrasound imaging," IEEE Trans. Ultrason., Ferroelect., Freq. Contr., pp. 275-282, July 1993.

[6] R. Mallart and M. Fink, "The van Cittert-Zernike theorem in pulse-echo measurements," J. Acoust. Soc. Amer., vol. 90, pp. 2718-2727, 1991. 